Rev. Int. Contam. Ambie. 35 (3) 713-722, 2019

DOI: 10.20937/RICA.2019.35.03.16

\title{
POTENCIAL DE RESIDUOS INDUSTRIALES GENERADOS EN CIUDAD JUÁREZ, CHIHUAHUA, MÉXICO, COMO COMBUSTIBLES ALTERNOS EN UN HORNO CEMENTERO
}

\author{
Potential of industrial wastes generated in Ciudad Juárez, Chihuahua, Mexico, as alternative fuels in a kiln
}

Antonia LUNA VELASCO, Luis Armando LOZOYA MÁRQUEZ y Guillermo GONZÁLEZ SÁNCHEZ*

Centro de Investigación en Materiales Avanzados, Av. Miguel de Cervantes 120, col. Complejo Industrial, 31136 Chihuahua, Chihuahua, México

* Autor para correspondencia: guillermo.gonzalez@cimav.edu.mx

(Recibido enero 2018; aceptado octubre 2018)

Palabras clave: residuos no reciclables, valorización energética, fracción residual orgánica

\section{RESUMEN}

En Ciudad Juárez, Chihuahua, México, se generan cantidades considerables de residuos sólidos no peligrosos, producidos principalmente por las industrias de manufactura, nogalera y maderera. El manejo integral de estos residuos requiere alternativas de tratamiento con mínimos impactos ambientales y económicos. Debido a que una fracción importante se compone de mezclas y materiales compuestos, el reciclaje no es viable. El aprovechamiento de la energía de estos residuos como combustibles alternos se considera una alternativa para su desecho. El propósito del estudio fue evaluar el potencial de los residuos sólidos industriales no reciclables generados en Ciudad Juárez, Chihuahua, como combustibles alternos para un horno cementero. En primer término, se clasificaron las diferentes corrientes de residuos y luego se caracterizaron física y químicamente de acuerdo con métodos estandarizados. Los residuos de biomasa comprendieron una fracción mayor como porcentaje de masa, seguidos por los categorizados como fracción residual orgánica de eficiencia energética (FROEE), que agrupan mezclas residuales de madera, papel y cartón, plásticos, y vestiduras automotrices. El poder calorífico inferior, ordenado de mayor a menor $(8869,5895$ y $3553 \mathrm{kcal} / \mathrm{kg})$, correspondió a los residuos de neumáticos, FROEE y biomasa, respectivamente. De los residuos FROEE, se identificó que el porcentaje en masa de cloro en poliuretano y algunas vestiduras automotrices era superior al límite establecido por los criterios internacionales de la industria cementera, por lo que se descartaron. La mezcla final de residuos FROEE, complementada con residuos de biomasa y de neumáticos, cumple con los parámetros críticos recomendados para uso como combustibles alternos; por tanto, las corrientes de residuos no reciclables pueden ser valorizadas energéticamente.

Key words: non-recyclable wastes, energetic valorization, residual organic fraction

\begin{abstract}
In Ciudad Juárez, Chihuahua, Mexico, high amounts of non-hazardous solid wastes are produced mainly by the manufacturing, nut processing and wood industries. Low-cost and environmental friendly alternatives are necessary to properly manage this kind of wastes. Recyclability is not feasible due to the predominance of waste
\end{abstract}


mixtures and composite residual materials. Energetic valorization of these wastes as alternative fuels is considered as a disposal alternative with both environmental and economic advantages. The objective of this study was to assess the potential of solid non-recyclable wastes produced in Ciudad Juárez, Chihuahua as alternative fuels in a kiln. First, the different categories of wastes were classified and then physically and chemically characterized based on standard methods. Biomass wastes (sawdust and nut shell) were the highest fraction regarding weight percent, followed by the wastes categorized as energetically efficient residual organic fraction (EEROF), which includes mixtures involving wood, carton, plastics and automotive fabrics. The lower heating values, from low to high $(8869,5895$ and $3553 \mathrm{kcal} / \mathrm{kg})$, corresponded to tires, EEROF and biomass wastes, respectively. The chlorine content among EEROF wastes, polyurethane plastic and some automotive fabrics was higher than the established limits for the cement industry, thus they were discarded. The final EEROF wastes formulation, supplemented with biomass wastes and tires, complies with the recommended values for critical parameters to be employed as alternative fuels, so these non-recyclable waste streams could be energetically valorized.

\section{INTRODUCCIÓN}

La generación de residuos sólidos urbanos (RSU) en México en 2015 fue de 53100000 t con una producción per cápita promedio diaria de $1.2 \mathrm{~kg}$ (SEMARNAT 2016a). En 2012, la región centro del país concentró el $51 \%$ de la generación de RSU, seguida por la región frontera norte con $16.4 \%$ y la Ciudad de México con el 11.8 \% (SEMARNAT 2016a). De 1997 a 2012, el patrón de generación de RSU se ha incrementado en las diferentes regiones, si bien con diferencias debidas al grado de desarrollo económico, medido como la contribución relativa de las entidades al producto interno bruto nacional. De esta manera, en el mismo periodo, el mayor aumento se presentó en la región frontera norte con un $214 \%$, pasando de una producción anual de $3153670 \mathrm{t} \mathrm{a} 6748850 \mathrm{t}$, seguido de la zona centro con un incremento del $53 \%$, la sur con $49 \%$ y la Ciudad de México con $20 \%$. El mayor aumento en la región frontera norte se debe a que los centros urbanos fronterizos han crecido de manera importante en las últimas décadas, principalmente por la instalación de plantas de ensamble y manufactureras. Sin embargo, la tasa de crecimiento de la infraestructura ambiental regional ha sido inferior a la económica, lo cual ha impactado negativamente en los recursos naturales, afectando el medio ambiente y la salud pública. Otro aspecto significativo es que la vecindad con EUA ha propiciado el intercambio de patrones culturales, afectando el modo de vida y el consumo de bienes, lo que también ha contribuido al incremento en la generación de residuos.

Aunque existe una extensa literatura sobre sistemas de manejo y caracterización de los residuos sólidos urbanos en México, ésta se ha concentrado principalmente en los residuos de origen domiciliario y un poco menos en los sectores comercial, de servicios y de instalaciones educativas (Durán-Moreno et al. 2013, Araiza-Aguilar et al. 2017). En cuanto a la caracterización y manejo de residuos sólidos no peligrosos generados por la industria, las entidades federativas se encargan prácticamente de los registros. De los generadores de residuos de manejo especial, el sector industrial tiene mayor área de oportunidad para cumplir una de las premisas fundamentales de la Ley General para la Prevención y Gestión Integral de los Residuos (SEMARNAT 2003), que es la valorización debida al volumen de residuos generados. Al determinar la generación anual promedio de residuos de manejo especial en nuestro país durante el periodo 2006-2012 (INECC 2012), se observó que la mayor cantidad correspondió a las excretas de ganado porcino y bovino lechero $(66710000 \mathrm{t})$, seguidas por papel y cartón (6 820000 t), y en tercer lugar los residuos de construcción y demolición (6 110000 t). Las dos primeras corrientes residuales pueden insertarse en sistemas de generación de energía a partir de residuos.

A diferencia de la recolección en el sector domiciliario, los residuos de manejo especial generados por la industria no se mezclan en su mayoría con compuestos orgánicos de alto contenido de humedad, lo cual representa una ventaja para asociarles un valor en la cadena económica (Rahman et al. 2015). La valorización de los residuos generados por el sector industrial se lleva a cabo mediante reciclado mecánico o químico para aquellas corrientes de materiales correctamente segregados (Ragaert et al. 2017), o bien mediante sistemas térmicos para las mezclas de materiales no susceptibles de reciclaje que aprovechan el valor energético de estos 
desechos (Lombardi et al. 2015). En la gestión de residuos sólidos, la valorización energética es una de las vías con mayor potencial real desde un punto de vista técnico, ecológico y económico (Ferreira et al. 2017). La valorización energética de residuos sólidos en un horno cementero se destaca por el impacto positivo al medio ambiente, como se ha reportado para la industria cementera en México (Güereca et al. 2015).

Por lo anterior, este estudio se enfocó al análisis del potencial de residuos sólidos industriales no reciclables generados en Ciudad Juárez, Chihuahua, México, como combustibles alternos en un horno cementero. Esto, a través de la determinación de la generación y caracterización de parámetros críticos recomendados por la industria cementera y criterios de la Asociación Europea Responsable de la Incineración y el Tratamiento de Residuos Especiales (EURITS, por sus siglas en inglés), para residuos con uso como combustibles alternos en un horno cementero (EC 2003, Madlool et al. 2011). Los residuos sólidos evaluados fueron principalmente de la industria manufacturera, nogalera y maderera, que comprenden los residuos de biomasa, neumáticos y los residuos categorizados como fracción residual orgánica de eficiencia energética (FROEE). Este último agrupa mezclas de residuos compuestas por madera, papel y cartón, plástico, así como textiles (naturales y sintéticos).

La generación de FROEE reportada en 2015 para la Ciudad de México fue de 89600 t (SEDEMA 2015), en tanto que el promedio anual de Ciudad Juárez reportado al gobierno estatal de Chihuahua en 2015 fue de 117100 t. La composición porcentual de las cuatro categorías de residuos FROEE fue muy similar en ambas ciudades: $72.40 \%$ para cartón y papel en la Ciudad de México frente a $73.56 \%$ en Ciudad Juárez; para madera fue de $12.68 \%$ en la Ciudad de México contra un 13.59 \% en Ciudad Juárez. En el caso de plásticos se tuvo un $13.24 \%$ en la Ciudad de México y en Ciudad Juárez un 12.22 \%. El aporte más bajo fue en textiles, con $1.68 \%$ en la Ciudad de México y $0.64 \%$ en Ciudad Juárez (fuente: base de datos inédita generada con el apoyo de la Secretaría de Desarrollo Urbano del estado de Chihuahua). Por lo tanto, Ciudad Juárez tiene un alto potencial para recuperación de corrientes residuales de alto valor energético, las cuales al presentarse mezcladas o como materiales compuestos no son susceptibles de reciclaje por el alto costo de separación.

El objetivo del presente estudio fue identificar corrientes de residuos generados por las empresas representativas de la manufactura automotriz y electrónica, así como de las industrias nogalera y maderera en Ciudad Juárez, Chihuahua, no aprovechables para reciclaje pero que pueden ser valorizados como combustibles alternos en horno cementero tubular giratorio. Esto, de conformidad con los criterios internacionales de la industria cementera con valores máximos para poder calorífico inferior y contenido de contaminantes como cloro y azufre expresados como porcentaje de masa.

\section{MATERIALES Y MÉTODOS}

\section{Muestreo de residuos y su origen}

Los residuos sólidos industriales no reciclables se obtuvieron de diversos sectores productivos de Ciudad Juárez (Chihuahua, México), particularmente de los que tenían una generación igual o superior a las 100 t/año, como la industria manufacturera automotriz, electrónica, nogalera y maderera. Las visitas se realizaron trimestralmente en el periodo comprendido entre septiembre de 2016 y agosto de 2017, para lo cual se contó con el apoyo de una empresa cementera. Se recolectaron diversas muestras de 5 a $10 \mathrm{~kg}$ a partir del método de cuarteo. El total de muestras fue de 41 individuales y 20 heterogéneas, tal y como las tenían almacenadas los diferentes generadores. Las corrientes de residuos obtenidas se clasificaron como biomasa (aserrín y cáscara de nuez), neumáticos y FROEE. Esta última agrupó a corrientes residuales de madera, papel, cartón, plásticos y vestiduras automotrices.

\section{Separación y molienda de residuos sólidos}

En el caso de las muestras compuestas por mezclas, se separaron los diferentes constituyentes de forma manual, y luego se pesaron para tener su masa referencial para la composición porcentual en masa de los residuos. Se tomaron $0.5 \mathrm{~kg}$ de todas las muestras de residuos (ya fueran en mezcla o individuales) para su molienda, la cual se realizó en un molino de corte Retsch Gmbh modelo SM 100 de $1.5 \mathrm{~kW}$ y velocidad de rotor de $2500 \mathrm{rpm}$. Todos los materiales se redujeron a gránulos de $0.25 \mathrm{~mm}$ para proceder a la caracterización de los diferentes parámetros físicos y químicos.

\section{Caracterización de los residuos}

La caracterización física y química de los residuos se hizo con base en los parámetros críticos recomendados para los residuos utilizados como combustibles alternos en horno cementero tubular rotatorio. Los parámetros medidos se describen a continuación. 
El poder calorífico se midió conforme a la norma ASTM D 5468 en un calorímetro de chaqueta sencilla Parr modelo 1341 con la bomba de oxígeno 1108CL, termómetro digital 6775 y bureta digital. La medición se hizo por duplicado en muestras simples y por triplicado en muestras compuestas por mezclas. Se determinó el porcentaje en masa del contenido de cloro siguiendo la norma ASTM D 4208, con un calorímetro de chaqueta sencilla Parr modelo 1341 con bomba de oxígeno $1108 \mathrm{CL}$, y medidor portátil multiparámetro Thermo Scientific Orion modelo STARA3295 con electrodo de ion selectivo de cloruros 9617BNWP. Los compuestos plásticos en los residuos se identificaron mediante un espectrómetro Perkin Elmer modelo Spectrum GX FT-IR System, con reflexión total atenuada (ATR, por sus siglas en inglés) marca Smiths, modelo Durasampl IR II con ventana de diamante de un rebote.

\section{Análisis proximal y elemental}

El contenido de cenizas y humedad se determinó como porcentaje en peso de acuerdo con las normas NMX-AA-18-1984 y NMX-AA-16-1984, respectivamente. Antes y después de las determinaciones, se obtuvieron los pesos residuales en una balanza analítica Sartorius Serie Competence CP225D. Las cenizas de los residuos se obtuvieron en una mufla 48000 Barnstead International/Thermolyne modelo Furnace F48020 y el contenido de humedad se determinó en un horno de secado Shell lab modelo CE3F. Respecto a la cuantificación de los diferentes metales pesados, se realizó conforme al método EPA $7000 \mathrm{~B}$ y las mediciones se llevaron a cabo en un espectrofotómetro de absorción atómica GBC modelo Avanta Sigma con generador de hidruros HG3000. Las muestras se digirieron en condiciones ácidas en parrilla de agitación/calentamiento, antes de la determinación de los metales $\mathrm{Cd}, \mathrm{Co}, \mathrm{Cu}, \mathrm{Mn}, \mathrm{Ni}, \mathrm{Pb}, \mathrm{Sb}$ y Tl, para lo cual se utilizó flama de acetileno-aire. Por su parte, para determinar Cr y V se utilizó flama de óxido nitroso-acetileno. En el caso del selenio, se empleó el generador de hidruros, en tanto que para Hg se utilizó vapor frío. Los límites de cuantificación del equipo se indican a continuación (en $\mathrm{mg} / \mathrm{kg}$ ): $\mathrm{Pb} \mathrm{0.1;} \mathrm{Cd} \mathrm{0.02;} \mathrm{Cr} \mathrm{0.2;} \mathrm{Mn} \mathrm{0.049;} \mathrm{Tl} \mathrm{0.5;} \mathrm{Co} \mathrm{0.1;}$ $\mathrm{Sb} 1 ; \mathrm{Cu} 0.06$; Ni 0.1; V 2; Hg 0.005, y Se 0.002.

También se determinó la composición elemental de carbono, hidrógeno, nitrógeno y azufre, la cual se obtuvo en porcentaje en peso mediante un analizador elemental CHNS-O Thermo Scientific modelo Flash Smart. La cantidad de oxígeno se obtuvo por diferencia en peso en relación con los otros elementos medidos.

\section{RESULTADOS Y DISCUSIÓN}

La caracterización de los componentes químicos en residuos sólidos es necesaria cuando se valoran como combustibles alternos. Los parámetros que se recomienda caracterizar en residuos con uso como combustibles alternos en horno cementero, de acuerdo a los criterios tanto de la Asociación Europea Responsable de la Incineración y el Tratamiento de Residuos Especiales (EURITS, por sus siglas en inglés) como de la industria cementera, son principalmente poder calorífico ( $>3343 \mathrm{kcal} / \mathrm{kg}$ ), contenido de cloro $(<0.2 \mathrm{y}<0.6 \%$ por planta cementera), azufre $(<2.5 \%)$, humedad $(<20 \%)$ y metales (en $\mathrm{mg} / \mathrm{kg}$ ) (totales < 2500; $\mathrm{Hg}<10 ; \mathrm{Cd}+\mathrm{Tl}+\mathrm{Hg}$ $<100$ (EC 2003, Madlool et al. 2011, Mokrzycki y Uliasz-Bochenczyk 2003).

Se llevó a cabo la caracterización de residuos industriales no reciclables generados por empresas representativas de los principales sectores de manufactura y transformación en Ciudad Juárez, clasificados como biomasa, neumáticos y FROEE. El primero se compone de biomasa de cáscara de nuez y aserrín de madera, mientras que el último se compone de varios tipos de plásticos, vestiduras de autos y cartón(Fig. 1). Estos residuos provienen de diferentes industrias de Ciudad Juárez con distintos giros, principalmente manufactura automotriz y electrónica, e industrias papelera, nogalera, maderera, etc. En el cuadro I se muestran los datos de caracterización de residuos industriales no reciclables generados por las empresas representativas de los principales sectores de manufactura y transformación en Ciudad Juárez. Puede observarse que los residuos de biomasa son los que se generan en mayor proporción, seguidos por los de tipo FROEE y neumáticos. La tendencia en la generación de distintos residuos industriales coincide con la actividad económica del estado de Chihuahua, que es el productor número uno de nuez (Reyes-Vázquez y Urrea-López 2016) y segundo en la producción de madera en México (SEMARNAT 2016b). Ambos procesos aportan gran cantidad de residuos de cáscara de nuez y aserrín de madera. Se estima que en 2015 se generaron entre 12700 y 17400 t de residuos de cáscara de nuez en Chihuahua, tomando en cuenta que en ese año se produjeron $79000 \mathrm{t}$ de nuez, de las cuales el $40 \%$ se vendió descascarada, y 40-50 \% del fruto corresponde a cáscara. En otro estudio se reportó que en Chihuahua se generan aproximadamente 201396 $\mathrm{t}$ de residuos de biomasa forestal (Moreno-López et al. 2017). Las manufacturas automotriz y aeroespacial también son relevantes en Ciudad Juárez, y por tanto generan parte importante de los residuos no 


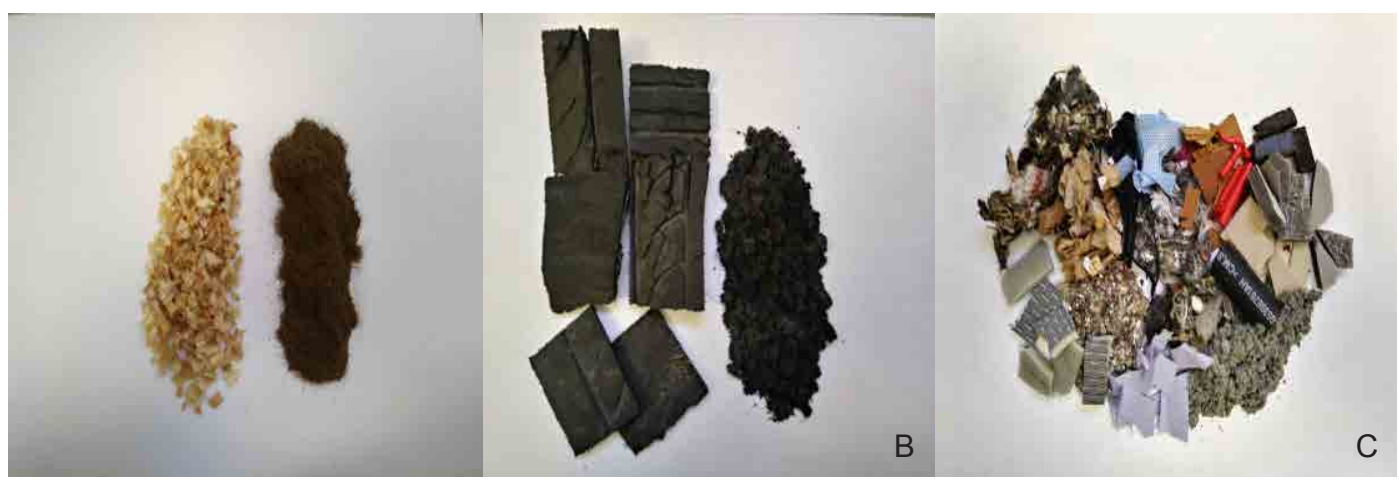

Fig. 1. Tipos de residuos industriales no reciclables generados en Ciudad Juárez, Chihuahua, que se caracterizaron para su valorización como combustibles alternos en horno cementero. (a) biomasa de aserrín y cáscara de nuez, (b) neumáticos y (c) mezcla de varios residuos plásticos, vestiduras de autos y cartón, clasificada como fracción residual orgánica de eficiencia energética (FROEE)

CUADRO I. VALORES DEL ANÁLISIS ELEMENTAL, PROXIMAL Y ENERGÉTICO DE RESIDUOS NO RECICLABLES Y NO PELIGROSOS GENERADOS EN CIUDAD JUÁREZ, CHIHUAHUA, AGRUPADOS COMO FROEE

\begin{tabular}{lcccccr}
\hline Componente \% en masa & \multicolumn{2}{c}{ Biomasa } & \multicolumn{2}{c}{ Neumáticos } & \multicolumn{2}{c}{ FROEE } \\
\hline Cloro & $0.15 \pm$ & 0.18 & $0.01 \pm$ & 0.00 & $4.92 \pm$ & 4.31 \\
Humedad & $8.80 \pm$ & 1.65 & $0.13 \pm$ & 0.04 & $2.50 \pm$ & 0.96 \\
Cenizas & $1.45 \pm$ & 0.60 & $2.73 \pm$ & 0.26 & $11.36 \pm$ & 14.84 \\
Carbono & $48.26 \pm$ & 1.11 & $82.8 \pm$ & \pm .7 & $58.95 \pm$ & 12.50 \\
Hidrógeno & $6.01 \pm$ & 0.13 & $7.6 \pm$ & \pm .5 & $8.47 \pm$ & 9.58 \\
Oxígeno & $43.08 \pm$ & 0.44 & $5.3 \pm$ & 0.2 & $26.15 \pm 22.97$ \\
Nitrógeno & $1.03 \pm$ & 1.37 & $0.6 \pm$ & \pm .1 & $3.21 \pm$ & 4.35 \\
Azufre & $0.02 \pm 0.03$ & $0.9 \pm$ & \pm .2 & $0.07 \pm$ & 0.06 \\
Poder calorífico (kcal/kg) & $3553 \pm 222$ & 8869 & \pm 139 & 5456 & \pm 857 \\
Generación en fracción & 0.63 & 0.17 & 0.20 \\
\hline
\end{tabular}

FROEE: residuos de fracción residual orgánica de eficiencia energética

reciclables tipo FROEE. De acuerdo con una base de datos de la Secretaría de Desarrollo Urbano y Ecología (SEDUE) del gobierno de Chihuahua, que concentra los datos reportados por las industrias generadoras de residuos, el promedio anual de residuos en Ciudad Juárez para el periodo 2009-2015 fue de 204600 t. Entre estos residuos, la categoría FROEE tiene la siguiente composición porcentual en masa: cartón y papel $(73.56 \%)$, madera $(13.59 \%)$ y plásticos (12.22\%) (fuente: base de datos inédita generada con el apoyo de la SEDUE de Chihuahua).

La disposición de los residuos genera costos e impactos ambientales, así como reducción del tiempo de vida útil de los rellenos sanitarios o sitios de disposición; por ello su valoración como combustibles alternos en horno cementero es una estrategia de interés tanto para los generadores como para las plantas cementeras, ya que se facilitaría la disposición y gestión de los residuos y a la vez se aprovecharía su poder calorífico de forma directa. De acuerdo con los resultados de caracterización (cuadro I), se observa que el poder calorífico de los tres tipos de residuos es superior al recomendado para combustibles alternos, correspondiendo el valor más alto a neumáticos seguidos por los residuos FROEE y la biomasa.

Los neumáticos se caracterizan por tener un alto poder calorífico. Kaantee et al. (2004) y Lamas et al. (2013) lo estimaron en 7500 a $8908 \mathrm{kcal} / \mathrm{kg}$, con un valor promedio de $8479 \mathrm{kcal} / \mathrm{kg}$ (Aranda-Usón et al. 2013), lo cual corresponde a lo establecido en el presente trabajo. Con relación a los residuos FROEE, gran aporte de su poder calorífico proviene de los componentes plásticos, ya que éstos en su mayoría aportan hasta $10748 \mathrm{kcal} / \mathrm{kg}$, que incluso se comparan con o sobrepasan el correspondiente a combustibles fósiles (Zhou et al. 2014). Aunque presentó el menor poder calorífico, la biomasa es adecuada para su uso como combustible alterno. El valor medido 
en biomasa es comparable con los reportados para biomasa de madera (3350 a $4300 \mathrm{kcal} / \mathrm{kg}$ ) y biomasa agrícola (4290 kcal/kg) (Raj et al. 2015).

Otro parámetro crítico en la valoración de residuos como combustibles alternos es el contenido de cloro. Los residuos de neumáticos y de biomasa cumplen con el valor recomendado para su uso como combustibles alternos; sin embargo, la mezcla de residuos FROEE sobrepasa ese valor, lo cual se atribuyó a alguno de sus componentes plásticos (poliuretanos) o vestiduras automotrices sintéticas que comúnmente contienen plásticos como vinilos policlorados. En reportes previos se indica que los plásticos con mayor contenido de cloro son los cloruros de polivinilo (PVC), los cuales contienen del 52 al $58 \%$ en peso de cloro (Zhou et al. 2014). En la literatura se establece que cuando se usan residuos con alto contenido de cloro como combustibles alternos en el horno cementero, el cloro se libera y debido a las condiciones del proceso reacciona con compuestos alcalinos como el $\mathrm{Ca}$, $\mathrm{Na}$ y $\mathrm{K}$, lo que provoca fenómenos de volatilización y condensación que desestabilizan el proceso (Enders y Haeseli 2011). En la planta cementera de interés, solamente se pueden utilizar residuos o mezclas de residuos como combustibles alternos con contenido de cloro $<$ $0.6 \%$ para mantener la estabilidad del proceso y las emisiones, por lo que fue importante identificar los compuestos que aportan cloro en la mezcla inicial de residuos FROEE, con el fin de descartarlos y obtener una mezcla adecuada.

Con relación al contenido de azufre, humedad y cenizas, están dentro de los valores recomendados para combustibles alternos en los tres tipos de residuos caracterizados. Solamente se destaca mayor contenido de $\mathrm{S}$ en neumáticos y mayor humedad en biomasa, cuyos valores son comparables con lo reportado en promedio para azufre en llantas $(1.54 \%)$ y humedad en biomasa agrícola (7.6\%) (Kaantee et al. 2004). El azufre implica problemas de emisión de contaminantes como $\mathrm{SO}_{\mathrm{x}}$ ( $\mathrm{Li}$ et al. 2009), en tanto que el aumento de humedad disminuye el poder calorífico liberado.

Del análisis elemental, el contenido de carbono es mayor en neumáticos, seguido de FROEE y biomasa, lo cual corresponde a la tendencia de poder calorífico en los residuos, ya que a mayor contenido de carbono mayor poder calorífico. El contenido de metales pesados también es un parámetro importante en los combustibles alternos, debido a que podrían incorporarse en el producto final o liberarse al medio ambiente a través de las emisiones de partículas. El contenido de metales totales fue de $85.1,255.1$ y $1342.28 \mathrm{mg} / \mathrm{kg}$ para los residuos de biomasa, neumáticos y mezcla de residuos FROEE, respectivamente. Con relación al contenido de mercurio se obtuvieron $0.01,1.51$ y $0.4 \mathrm{mg} / \mathrm{kg}$ en residuos de biomasa, neumáticos y mezcla de residuos FROEE, respectivamente. Los metales cadmio y talio no se detectaron en ninguna de las muestras de residuos, por lo que la sumatoria de $\mathrm{Cd}, \mathrm{Tl}$ y $\mathrm{Hg}$ sería igual al contenido de Hg. Cabe notar que los valores de metales pesados detectados en los residuos son menores a los recomendados para residuos como combustibles alternos por los criterios internacionales EURITS (en $\mathrm{mg} / \mathrm{kg}$, metales totales $<2500 ; \mathrm{Hg}<10 ; \mathrm{Cd}+\mathrm{Tl}+\mathrm{Hg}<100)$, por lo que no representarían un problema en el coprocesamiento de los residuos en el horno cementero.

En un estudio previo se evaluaron diferentes tipos de residuos como combustibles alternos en la producción de cemento, los cuales presentaron variaciones importantes en el contenido de metales. Entre éstos se incluyeron plásticos y una mezcla con 12 corrientes diferentes de residuos. En ambos casos, el contenido de metales estuvo dentro de lo recomendado y se observó que no aportó efectos negativos, tanto en emisiones de partículas como en calidad del producto durante su coprocesamiento en tasas de sustitución del combustible convencional del 20.6 y $9.6 \%$, respectvamente (Parlikar et al. 2016).

Después de este análisis, se estableció que la mayoría de los residuos evaluados cumplen con todos los parámetros críticos para combustibles alternos en horno cementero. Solamente en la mezcla inicial de residuos FROEE se observó que el cloro estaba por arriba de lo recomendado, lo cual se atribuyó a alguno de sus componentes plásticos, así como a vestiduras automotrices. Por ello fue importante clasificar e identificar los componentes plásticos que conforman este tipo de residuos, así como caracterizar los parámetros críticos de cada componente plástico, particularmente su contenido de cloro, al igual que en residuos de vestiduras automotrices y cartón. Esto con el fin de descartar los residuos con alto contenido de cloro y obtener una mezcla FROEE adecuada para uso como combustibles alternos en el horno cementero.

Primero, se separaron y clasificaron los componentes de la mezcla de residuos FROEE y los componentes plásticos se caracterizaron mediante espectroscopia de infrarrojo. En la figura 2 se muestran los principales plásticos identificados con este método, así como el contenido de cloro y poder calorífico de los diferentes plásticos, vestiduras automotrices y cartón. Como referencia, también se incluyen los datos correspondientes de un combustible convencional, el carbón mineral. En la mezcla 


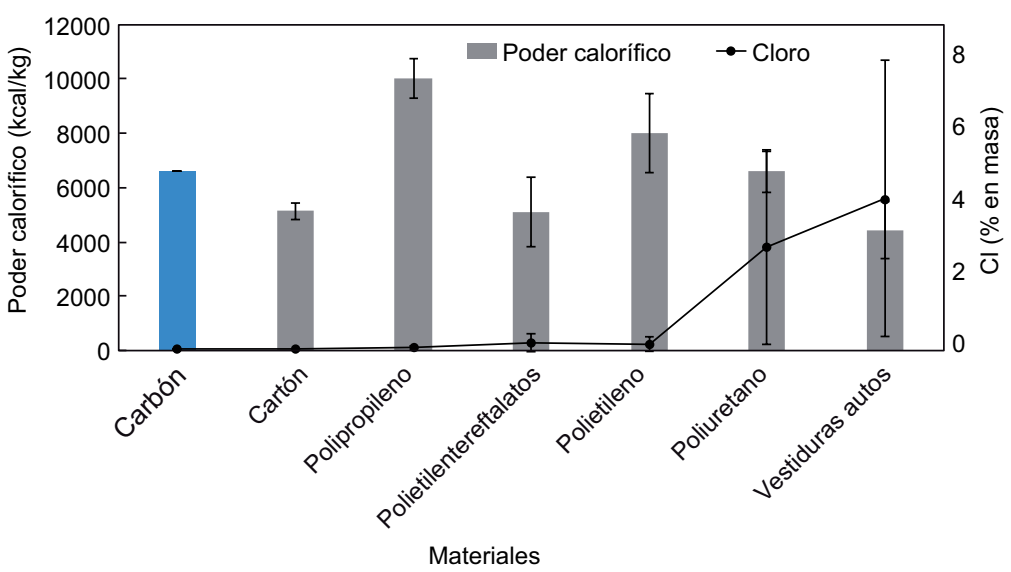

Fig. 2. Contenido de cloro y poder calorífico de los componentes de la mezcla inicial de residuos FROEE: cartón, diferentes tipos de plásticos y vestiduras automotrices. Como referencia se incluyen los datos del combustible fósil convencional, carbón mineral

de residuos FROEE se identificaron cuatro tipos de plásticos: polietilentereftalato (PET), polipropileno (PP), polietileno (PE) y poliuretano (PU), de los cuales los primeros tres están dentro de los cinco más utilizados en la industria (Zhou et al. 2014). En dicha figura también se observa claramente que el residuo plástico identificado como PU, tiene gran aporte de cloro equivalente en promedio a $2.8 \%$ en peso. El PU no contiene cloro en su estructura química, por lo que la presencia de cloro en este residuo se puede atribuir a algún aditivo clorado o bien al posible contacto de estos residuos con compuestos clorados. Cabe aclarar que los valores de cloro representan el promedio de diferentes poliuretanos presentes en la mezcla de residuos FROEE y la desviación estándar alta indica una gran variación del contenido de cloro entre un tipo de poliuretano y otro. Algunas vestiduras automotrices también presentaron alto contenido de cloro $(4.2 \pm 3.8 \%)$, lo cual se debe a la presencia polivinilos clorados, como el cloruro de polivinilo identificado por espectroscopia de infrarrojo. De lo anterior se concluye que los residuos descartables en la mezcla de FROEE son el PU y vestiduras automotrices con alto contenido de cloro.

En cuanto al poder calorífico, los plásticos identificados en los residuos FROEE presentaron excelente poder calorífico e incluso el PP, PU y PE tienen un poder calorífico mayor al del carbón mineral, que es alrededor de $6600 \mathrm{kcal} / \mathrm{kg}$ (Kaantee et al. 2004). Los valores de poder calorífico obtenidos concuerdan con lo reportado previamente para ese tipo de plásticos (Zhou et al. 2014). Por su parte los residuos de cartón caracterizados tienen buen poder calorífico y muy bajo contenido de cloro, lo que coincide con lo reportado en un trabajo previo (Zhou et al. 2014). Respecto al contenido de metales totales y mercurio, estos son muy inferiores a lo recomendado en cada uno de los residuos que conforman la FROEE (Fig. 3), lo que es consistente con el contenido de metales pesados totales determinado en la mezcla de residuos FROEE. No se detectaron trazas de cadmio y talio en ninguna de las muestras de residuos, por lo que la sumatoria de $\mathrm{Cd}, \mathrm{Tl}$ y $\mathrm{Hg}$ sería igual al contenido de $\mathrm{Hg}$.

De acuerdo con la caracterización de cada uno de los componentes de la mezcla inicial de residuos FROEE, se obtuvo que éstos tienen excelente poder calorífico y que se deben descartar los componentes plásticos identificados con alto contenido de cloro, como el PU y las vestiduras automotrices con alto contenido de cloro para obtener una mezcla adecuada para uso como combustible alterno, en este caso con un contenido de cloro $<0.6 \%$. Por lo anterior se obtuvo una mezcla de residuos FROEE final que se caracterizó física y químicamente para corroborar que cumpliera con los parámetros críticos. En el cuadro II se muestran los valores del análisis físico y químico de la mezcla final de residuos FROEE. En ellos se observa que el contenido de cloro es inferior al valor recomendado por los criterios internacionales EURITS $(<0.2 \%)$, lo cual corrobora que los residuos descartados (PU y vestiduras con alto contenido de cloro) aportaban el cloro en la mezcla FROEE inicial. Asimismo, el poder calorífico de la mezcla final de residuos FROEE se incrementó ligeramente, lo cual era esperado, ya que el PU y las vestiduras tienen menor poder calorífico en comparación con los otros componentes plásticos en la mezcla. El contenido 


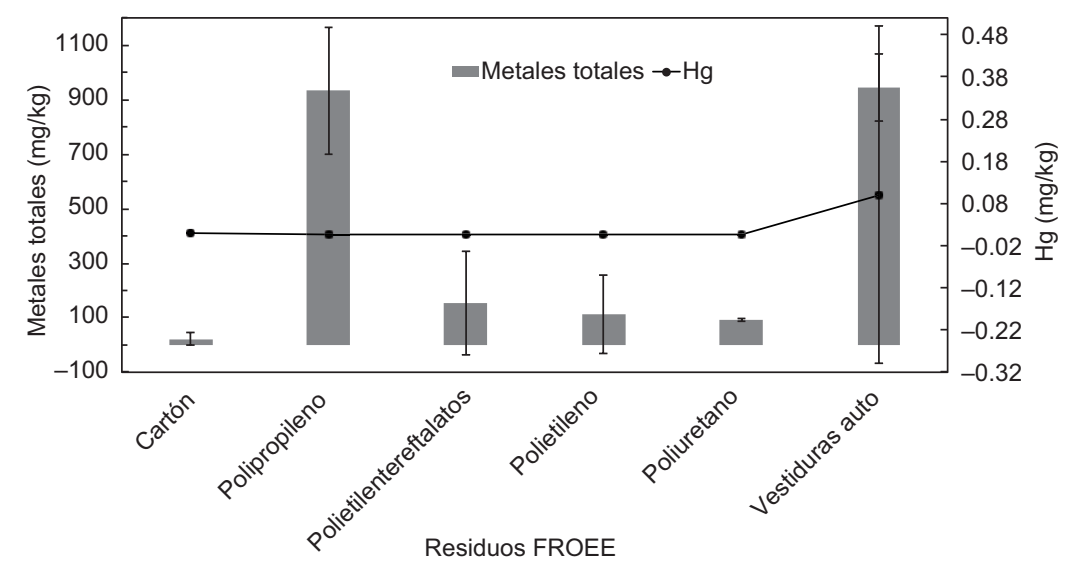

Fig. 3. Contenido de metales totales y mercurio en los componentes de la mezcla de residuos FROEE: vestiduras automotrices, cartón y diferentes tipos identificados de plásticos. Los valores de metales recomendados para residuos como combustibles alternos por criterios de la Asociación Europea Responsable de la Incineración y el Tratamiento de Residuos Especiales (EURITS), son (en $\mathrm{mg} / \mathrm{kg}$ ): metales totales $<2500, \mathrm{Hg}<10, \mathrm{Cd}+\mathrm{Tl}+\mathrm{Hg}<100$

CUADRO II. VALORES DEL ANÁLISIS ELEMENTAL, PROXIMAL Y ENERGÉTICO DE LA MEZCLA FINAL DE RESIDUOS FROEE GENERADOS EN CIUDAD JUÁREZ, CHIHUAHUA

\begin{tabular}{lc}
\hline Componente \% en masa & FROEE \\
\hline Cloro & $0.13 \pm 0.13$ \\
Humedad & $2.83 \pm 0.79$ \\
Cenizas & $2.85 \pm 1.32$ \\
Carbono & $64.02 \pm 12.59$ \\
Hidrógeno & $12.7 \pm 8.7$ \\
Oxígeno & $17.7 \pm 25.02$ \\
Nitrógeno & $4.63 \pm 5.08$ \\
Azufre & $0.06 \pm 0.08$ \\
Poder calorífico $(\mathrm{kcal} / \mathrm{kg})$ & $5895 \pm 82$ \\
\hline
\end{tabular}

\begin{tabular}{lr}
\hline \multicolumn{2}{c}{ Metales $(\mathrm{mg} / \mathrm{kg})$} \\
\hline Totales & $56.32 \pm 36.40$ \\
Mercurio & $2.74 \pm 1.93$ \\
$\mathrm{Cd}+\mathrm{Tl}+\mathrm{Hg}$ & $2.74 \pm 1.93$ \\
\hline
\end{tabular}

FROEE: residuos de fracción residual orgánica de eficiencia energética

de metales totales y $\mathrm{Hg}$ también disminuyó en la mezcla FROEE final, ya que se eliminaron vestiduras automotrices, las cuales son uno de los residuos con mayor aporte de metales.

Como resultado de la clasificación, identificación y caracterización de los componentes de la mezcla inicial de residuos FROEE, se obtuvo una mezcla de residuos que cumple con los parámetros críticos recomendados por los criterios EURITS y de la industria cementera, para su uso como combustibles alternos en horno cementero. La mezcla obtenida de residuos FROEE se compone de corrientes de residuos de cartón, plásticos e incluso vestiduras automotrices con contenido de cloro menor al valor límite establecido. En estudios y desarrollos previos se han reportado procesos de clasificación y eliminación de residuos como estrategias para controlar parámetros críticos en residuos con potencial como combustibles alternos, particularmente en aquellos con alto contenido de cloro como los plásticos (Yuliang et al. 2015). También se han desarrollado mezclas de diferentes corrientes de residuos para que cumplan con los parámetros críticos; en particular se han reportado mezclas de residuos plásticos con diferentes tipos de biomasa (Nafid et al. 2008, Richards y Agranovski 2015, Parlikar et al. 2016). El uso frecuente de biomasa como combustible alterno en los hornos cementeros se debe a que este tipo de residuos normalmente se generan en abundancia en sitios aledaños; sólo varía el tipo de biomasa por región, y por tanto sus características físicas y químicas (Aranda-Usón et al. 2013). Los residuos de biomasa fueron los que tuvieron mayor disponibilidad en Ciudad Juárez, por lo que se cuenta con cantidades suficientes para utilizarlos en una fracción considerable como combustibles alternos en el horno cementero. Sólo hay que tomar en cuenta que el valor recomendado de sustitución de combustible convencional por biomasa es de aproximadamente el $20 \%$ como máximo, para mantener estables las condiciones del proceso $(\mathrm{Mu}-$ rray y Price 2008). Por consiguiente, los residuos de 
biomasa (cáscara de nuez y aserrín) se pueden utilizar en fracciones adecuadas combinadas con los residuos de neumáticos y la mezcla final de residuos FROEE, como combustibles alternos en horno cementero. De esta manera se pueden alcanzar niveles de sustitución de combustible convencional, de hasta un $45 \%$, con contenidos de cloro, azufre, humedad y cenizas por debajo de los límites establecidos por la industria cementera.

\section{CONCLUSIONES}

Los residuos industriales no reciclables generados en Ciudad Juárez, Chihuahua, disponibles para su uso como combustibles alternos, constan de una fracción mayor de biomasa de cáscara de nuez y aserrín, seguida por los residuos clasificados como FROEE y los neumáticos. Los residuos FROEE se componen de cuatro tipos de plásticos diferentes, vestiduras automotrices y cartón. Como resultado de la clasificación, identificación y caracterización de todos los residuos se estableció que tanto los residuos de biomasa como los de neumáticos cumplen con los parámetros críticos recomendados por los criterios internacionales EURITS y la industria cementera, para su uso como combustibles alternos en el horno cementero. Asimismo, se obtuvo una mezcla de residuos FROEE que cumple con determinados parámetros. De las corrientes de residuos se identificó que el poliuretano y algunas vestiduras automotrices contienen alto contenido de cloro, por lo que se descartaron para su uso como combustibles alternos. En general, se concluye que los residuos sólidos industriales no reciclables generados en Ciudad Juárez por las empresas representativas de los principales sectores de manufactura y transformación, son apropiados para aprovechar su poder energético como combustibles alternos en horno cementero en condiciones estables del proceso. En un siguiente estudio se establecerá la correlación entre el porcentaje de mezcla residual quemada como combustible alterno en horno cementero tubular rotatorio y el nivel de emisiones de contaminantes a la atmósfera.

\section{REFERENCIAS}

Araiza-Aguilar J.A., Chávez Moreno J.C. y Moreno Pérez J.A. (2017). Cuantificación de residuos sólidos urbanos generados en la cabecera municipal de Berriozábal, Chiapas, México. Rev. Int. Contam. Ambie. 33 (4), 691-699. DOI: 10.20937/RICA.2017.33.04.12
Aranda-Usón A., López-Sabirón A.M., Ferreira G. y LleraSastresa E. (2013). Uses of alternative fuels and raw materials in the cement industry as sustainable waste management options. Renew. Sust. Energ. Rev. 23, 242-260.

DOI: $10.1016 /$ j.rser.2013.02.024

Durán-Moreno A., Garcés Rodríguez M., Velasco A.R., Marín Enríquez J.C., Gutiérrez Lara R., Moreno Gutiérrez A. y Delgadillo Hernández N.A. (2013). Mexico City’s municipal solid waste characteristics and composition analysis. Rev. Int. Contam. Ambie. 29 (1), 39-46.

Enders M. y Haeseli U. (2011). Reactions of alkalis, chlorine and sulfur during Clinker production. Cement Int. 9, 38-53.

EC (2003). Refuse derived fuel, current practice and perspectives. Report B4-3040/2000/306517/MAR/ E3. Directorate General for Environment, European Commission, Wiltshire, Gran Bretaña, 229 pp.

Ferreira S., Cabral M., da Cruz N.F., Simoes P. y Marques R.C. (2017). The costs and benefits of packaging waste management systems in Europe: the perspective of local authorities. J. Environ. Plan. Manag. 60 (5), 773791. DOI: 10.1080/09640568.2016.1181609

Güereca L. P., Torres N. y Juárez-López C.R. (2015). The co-processing of municipal waste in a cement kiln in Mexico. A life-cycle assessment approach. J. Clean Prod. 107, 741-748.

DOI: $10.1016 /$ j.jclepro.2015.05.085

INECC (2012). Diagnóstico básico para la gestión integral de los residuos 2012. Instituto Nacional de Ecología y Cambio Climático, Ciudad de México, México, 201 pp.

Kaantee U., Zevenhoven R., Backman R. y Hupa M. (2004). Cement manufacturing using alternative fuels and the advantages of process modelling. Fuel Process. Technol. 85 (4), 293-301.

DOI: 10.1016/S0378-3820(03)00203-0

Lamas W.D.Q., Palau J.C.F. y Camargo J.R.D. (2013). Waste materials co-processing in cement industry: Ecological efficiency of waste reuse. Renew. Sust. Energ. Rev. 19, 200-207. DOI: 10.1016/j.rser.2012.11.015

Li P.S., Hu Y., Yu W., Yue Y.N. Xu, Q., Hu S., Hu N.S. y Yang J. (2009). Investigation of sulfur forms and transformation during the co-combustion of sewage sludge and coal using X-ray photoelectron spectroscopy. J. Hazard. Mater. 167 (1-3), 1126-1132. DOI: 10.1016/j.jhazmat.2009.01.115

Lombardi L., Carnevale E. y Corti A. (2015). A review of technologies and performances of thermal treatment systems for energy recovery from waste. Waste Manage. $37,26-44$.

DOI: $10.1016 /$ j.wasman.2014.11.010 
Madlool N.A., Saidur R., Hossain M.S. y Rahim N.A. (2011). A critical review on energy use and savings in the cement industries. Renew. Sust. Energy. Rev. 15 (4), 2042-2060. DOI: 10.1016/j.rser.2011.01.005

Mokrzycki E. y Uliasz-Bochenczyk A. (2003). Alternative fuels for the cement industry. Appl. Energy 74 (1-2), 95-100. DOI: 10.1016/S0306-2619(02)00135-6

Moreno-López M., Alarcón-Herrera M.T. y MartínDomínguez I.R. (2017). Feasibility of pelletizing forest residues in Northern Mexico. Waste Biomass Valor. 8 (3), 923-932. DOI: 10.1007/s12649-016-9623-0

Murray A. y Price L. (2008). Use of alternative fuels in cement manufacture: analysis of fuel characteristics and feasibility for use in the Chinese cement sector. LBNL-525E. Ernest Orlando Lawrence Berkeley National Laboratory, US-EPA, 63 pp.

Nafid M., Koekkoek R., Der Linden A.M.G., Schoen L.A.A. y Schristoffel A.R. (2008). Improved combustibility of a waste plastic based fuel source. Patente núm. WO2008107042A2. OMPI, Organización de las Naciones Unidas, Ginebra, Suiza.

Parlikar U., Bundela P.S., Baidya R., Ghosh S.Kr.y Ghosh S. K. (2016). Effect of variation in the chemical constituents of wastes on the co-processing performance of the cement kilns. Procedia Environ. Sci. 35, 506-512. DOI: 10.1016/j.proenv.2016.07.035

Ragaert K., Delva L. y van Geem K. (2017). Mechanical and chemical recycling of solid plastic waste. Waste Manage. 69, 24-58.

DOI: 10.1016/j.wasman.2017.07.044

Rahman A., Rasul M.G., Khan M.M.K. y Sharma S. (2015). Recent development on the uses of alternative fuels in cement manufacturing process. Fuel 145, 8499. DOI: 10.1016/j.fuel.2014.12.029

Raj T., Kapoor M., Gaur R., Christopher J., Lamba B., Tuli D.K. y Kumar R. (2015). Physical and chemical characterization of various Indian agriculture residues for biofuel production. Energy Fuels 29 (5), 3111-3118. DOI: $10.1021 /$ ef5027373
Reyes-Vázquez N.C. y Urrea-López R. (2016). Retos y oportunidades para el aprovechamiento de la nuez pecanera en México. Centro de Investigación y Asistencia en Tecnología y Diseño del Estado de Jalisco, Guadalajara, México, 111 pp.

Richards G. y Agranovski I.E. (2015). Air emission from the co-combustion of alternative derived fuels within cement plants: Gaseous pollutants. J. Air Waste Manage. Assoc. 65 (2), 186-196.

DOI: $10.1080 / 10962247.2014 .984084$

SEDEMA (2015). Inventario de residuos sólidos de ciudad de México. Secretaría de Medio Ambiente, Gobierno de la Ciudad de México, 114 pp.

SEMARNAT (2003). Ley general para la prevención y gestión integral de los residuos. Secretaría de Medio Ambiente y Recursos Naturales. Diario Oficial de la Federación, 8 de octubre.

SEMARNAT (2016a). Informe de la situación del medio ambiente en México 2015. Compendio de estadísticas ambientales. Indicadores clave, de desempeño ambiental y de crecimiento verde. Resumen ejecutivo. Secretaría de Medio Ambiente y Recursos Naturales, Ciudad de México, 472 pp.

SEMARNAT (2016b). Anuario estadístico de la producción forestal. Dirección General de Gestión Forestal y de Suelos, Secretaría de Medio Ambiente y Recursos Naturales, Ciudad de México,. 225 pp.

Yuliang C., Xuequan Y., Jiong P., Dongbo Z. y Jing X.M. (2005). Process for treating urban house refuse using dry method cement production system. China, patente No. CN1623698A, 8 de junio.

Zhou H., Meng A., Long Y., Li Q. y Zhang Y. (2014). Classification and comparison of municipal solid waste based on thermochemical characteristics. J. Air Waste Manage. Assoc. 64 (5), 597-616.

DOI: $10.1080 / 10962247.2013 .873094$ 